\title{
Integration of ontology and knowledge-based optimization in process synthesis applications
}

\author{
Dr. Franjo Cecelja ${ }^{\mathrm{a}}$, Prof. Antonis Kokossis ${ }^{\mathrm{b}}$, Dr. Du Du ${ }^{\mathrm{a}}$, \\ ${ }^{a}$ Process \& Information Systems Engineering, FEPS, University of Surrey, Guildford, \\ U.K \\ ${ }^{b}$ School of Chemical Engineering, National Technical University of Athens, Zografou \\ Campus, Athens, Greece
}

\begin{abstract}
Previous research has shown that knowledge-based optimization models in process synthesis applications are more robust in both providing final outputs and improving computational performance. This expands this approach by implementing a general knowledge models which in turn enables interpretation of solutions so that non-experts understand detailed procedures of optimization. To this end, an automatic ontologybased optimization system that links rule-based optimization model and ontology has been introduced for the purpose to both improve optimization performance and to present new extracted knowledge at optimization run-time. A benchmark reactor network design synthesis case is studied for comparison of performance. The concomitant results show that not only can ontology-based optimization system improve robustness of solutions and computational performance, but also it enables a more accurate understanding of the process synthesis procedures and presents extracted knowledge in a decent format.
\end{abstract}

Keywords: stochastic optimization, knowledge models, ontology, process synthesis, high throughput.

\section{Introduction}

An extensive use of both deterministic and stochastic optimization algorithms to solve reactor network design synthesis problems have been reported (Achenie and Biegler, 1990; Kokossis and Floudas, 1990; Marcoulaki and Kokossis, 1996; Mehta and Kokossis, 2000; Yang, Linke and Kokossis, 2006). To improve computational performance, recent research effort was directed towards the use of computer grid techniques with Tabu Search algorithm (Du, Yang, Kokossis and Linke, 2007) and Simulated Annealing (SA) and Cascade algorithm (CA) (Yang, 2009; Du, 2009). In addition, knowledge-based optimization models have been applied in process synthesis experiments (Labrador-Darder, 2009; Du, Cecelja and Kokossis, 2009). It has been shown that the knowledge-based optimization model on grids accelerates optimization process as it guides optimization search towards promising regions with the assist of production rules (Du, 2009). Although they have shown robustness in achieving final solution and computational performance, none of these approaches have yet been upgraded to general knowledge level and interpretation in human languages.

This paper presents an ontology based synthesis approach that was designed for the extraction, interpretation and exploitation of design knowledge in process synthesis and combines stochastic optimization algorithm, ontologies and analytical tools. Defined as ...an explicit specification of a conceptualization (Gruber, 1993), the ontology is an attractive formalism for knowledge modeling in two distinct representation 
environments: frames and XML based languages such as OWL (Ontology Web Language). The knowledge model here is based on OWL representation using all four components: classes which represent concepts in the domain, instances (or individuals) which represent the objects of a domain, properties which are attributes of classes and relationships between them, and restrictions which express constraints on the values of properties. A commercially available inference engine RaserPro is used for ontology classification and hence inferring selective knowledge.

\section{Ontology-supported optimization model}

Figure 1 shows the proposed knowledge-based optimization model with Cascade Algorithm (CA) (Yang, 2009; Du, 2009). In general terms, the idea of the CA is to split a single Markov chain normally used with Simulated Annealing algorithm into sections and submit these sections for parallel processing by different workers. The objective values of the solutions are stored into the pools together with the concomitant intrinsic parameters which are stored in associated partitions, all under the control of the optimization server. In the case of optimizing reactor networks presented in superstructure format (Du, Cecelja and Kokossis, 2009) and which is used for verification, the intrinsic parameters include number of reactors, reactor types, reactor sequence, reactor volumes, feed flow, split fraction, concentration of desired product (objective value) etc. The optimization server selects a temperature and a solution in the pool that is associated with the temperature based on a group of pre-defined production rules (IF conditions, THEN actions) for each of the workers in the computer grid to execute one section of SA Markov chain (Du, Cecelja and Kokossis, 2009). For example, if the percentage of promising solutions with one type of reactor sequence is found to be higher than all the others, then we focus the optimization search towards the regions that can generate solutions with this kind of reactor sequence. In general terms, the purpose of applying production rules on solution selection is to classify solutions by the patterns of intrinsic parameters and to guide optimization search towards promising regions where more high quality solutions are found, hence accelerating the convergence. The termination criterion of the knowledge-based optimization model is determined by the frequency of the number of solutions in the lowest pool (Yang, 2009).

The whole process is illustrated in Figure 1 where $c$ is the total number of workers identified as $W_{l}, W_{2}, W_{3}, \ldots, W_{c}$. New generated solutions $S_{k}^{\prime}, k \in(1,2, \ldots, c)$ are sent to the pools $P_{l}, P_{2}, \ldots, P_{w}$, with each one being associated with the temperature $T_{j}$, $j \in(1,2, \ldots, w) . S_{k}^{s}$ is selected initial solution from pool temperature $T_{k}^{s}$ for worker $W_{k}$. Both objective values and intrinsic parameters of solutions are populated continuously during the optimization process and analyzed and new knowledge based on intrinsic parameters are updated in ontology. By interpreting the extracted knowledge to formulate production rules, actions (biased search) are taken for next few sections of SA Markov chains until new production rules are formulated. In order to make the whole process automated, a java base agent is added to interface the knowledge-based optimization model with ontology. The operation of the agent is as follows:

1. Populate ontology with intrinsic data of solutions from the optimization server continuously;

2. Analyze the solution data statistically, such as calculating the percentage of promising solutions with parameters (e.g. reactor sequences) and present the results 
by adding an instance about these percentages in ontology between arrival of new solutions;

3. Infer ontology to generate new knowledge, e.g. preferred reactor sequence;

4. Formulating new rules for changing search direction for next few sections of Markov chain;

5. Check if termination criterion is met: if yes, stop the process, otherwise go back to Step 1 for the next loop.

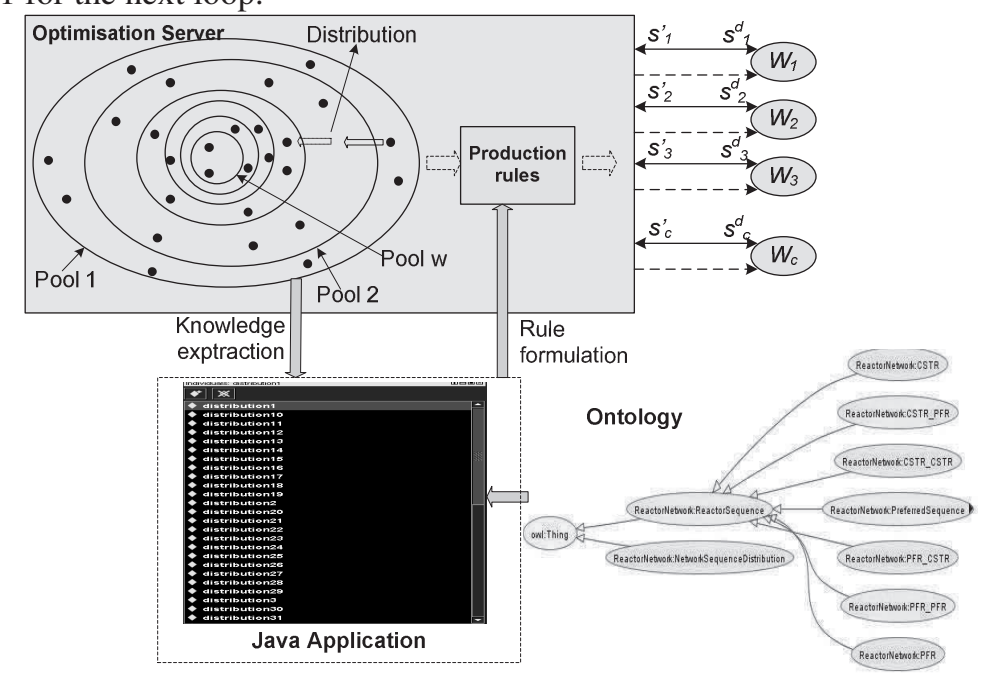

Figure 1 An ontology-supported optimization model

\section{Results}

3.1. An ontology for an intrinsic parameter - reactor sequence

In this paper the superstructure representation of reactor network is selected to verify the proposed optimization concept with intrinsic parameters which include the number of active reactors (up to 4), two reactor types (CSTR - continuous stirred tank reactor and PFR - plug flow reactor), reactor sequence, single/multiple feeds, reactor volumes, and volumes passed through bypasses and recycles (Mehta and Kokossis, 2000; Yang, Linke and Kokossis, 2006). The aim of optimization with superstructure model is to find out a combination of all these parameters, which can generate the maximum concentration of desired products with minimal complexity of the structure. Figure 1 shows a part of reactor network ontology which presents reactor sequence of the $1^{\text {st }}$ reactor $r_{1}$ and $2^{\text {nd }}$ reactor $r_{2}$ (ReactorSequence class), and percentages of number (e.g. 20) of top solutions with each $r_{1}+r_{2}$ sequence by objective values described as instances (distributionX, $\quad X \in(1,2,3, \ldots)$ ) of NetworkSequenceDistribution class. Since $r_{1}, r_{2} \in(C S T R, P F R)$, there are maximum 6 possible sequence combinations of $r_{l}+r_{2}$ which are: 1) CSTR, 2) PFR, 3) CSTR+CSTR, 4) CSTR+PFR, 5) PFR+CSTR, and 6) PFR+PFR, which correspond to CSTR, PFR, CSTR_CSTR, CSTR_PFR, PFR_CSTR, $P F R \_P F R$ ontology classes, respectively. Thus, each of distributionX instances has 6 datatype properties - hasCPercentage, hasPPercentage, hasCCPercentage, hasCPPercentage, hasPCPercentage and hasPPPercentage, which respectively show the percentage of CSTR, PFR, CSTR+CSTR, CSTR+PFR, PFR+CSTR and PFR+PFR 
solutions among the top solutions ordered by their objective values. PreferredSequence class is used to show the $r_{1}+r_{2}$ sequence with the highest percentage from top solutions at optimization run-time.

\subsection{A case study of Van de Vusse application}

Extensively studied application known as Van de Vusse, for which global solution has not been claimed so far, is chosen to test the ontology-supported optimization (Achenie and Biegler, 1990; Kokossis and Floudas, 1994; Mehta and Kokossis, 1998).

First to examine is the impact of the sequence of the $1^{\text {st }}$ reactor $r_{1}$ and $2^{\text {nd }}$ reactor $r_{2}$ on the optimization performance. With optimization search progressing, the values for $r_{I}$ and $r_{2}$ of a fixed number of top best solutions ordered by objective value, e.g. 20, are collected continuously every 10 seconds. The collected data from top solutions are statistically analyzed and percentages of the solutions with different $r_{l}+r_{2}$ sequences are calculated. Then, a new instance distributionX is added to ReactorSequenceDistribution class to present the percentages of appearance of each $r_{1}+r_{2}$ sequence in current top solutions, as shown in Figure 2. Note that has $r_{1} / r_{1} r_{2}$ Percentage are data properties that present the percentage of the solutions with $r_{l}$ or $r_{l}+r_{2}$ sequence and $\mathrm{C}$ and $\mathrm{P}$ indentify CSTR and PFR reactors, respectively. For example, hasCPPercentage means the percentage of the solutions with CSTR as the $1^{\text {st }}$ reactor and PFR as the $2^{\text {nd }}$ reactor. By comparing the percentages of solutions with each combination of $r_{1}$ and $r_{2}$, the highest percentage is selected and the relevant reactor sequence $r_{l}+r_{2}$ is considered as a promising solution, hence strengthening the optimization towards the promising regions. For the Van de Vusse case, 55\% the best solutions have CSTR+PFR sequence at the run-time. Thus, optimization search is directed in the region with solutions taking CSTR as the $1^{\text {st }}$ reactor $r_{1}$ and PFR as the $2^{\text {nd }}$ reactor $r_{2}$ by changing search direction for next few sections of SA Markov chain.

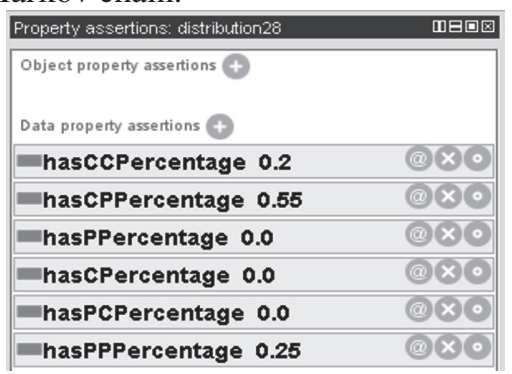

Figure $2 \mathrm{An}$ instance of the percentage of top solutions with different combinations of $r_{1}$ and $r_{2}$

Figure 3 shows the percentages of promising solutions with different $r_{l}+r_{2}$ sequences that are presented by distributionX instances during the optimization process. As shown, for Van de Vusse application, 55\% of the top solutions have CSTR+PFR sequence at the beginning of the optimization search, which leads optimization search towards CSTR+PFR directions. Then the percentage of PFR+PFR solutions (from $35 \%$ to $40 \%$ to $50 \%$ ) increases and exceeds the percentage of CSTR+PFR top solutions. Consequently, the optimization search direction is adjusted towards PFR+PFR regions. With the optimization search progressing, CSTR+PFR solutions become dominant in the promising solutions again, with a percentage of $70 \%$ at the termination point.

The comparison of the whole optimization of Van de Vusse case using ontology based knowledge based model and without is shown in Table 1 and clearly indicates more robust and faster convergence. Note that the values in Table 1 are average values from repeated 10 experiments. 


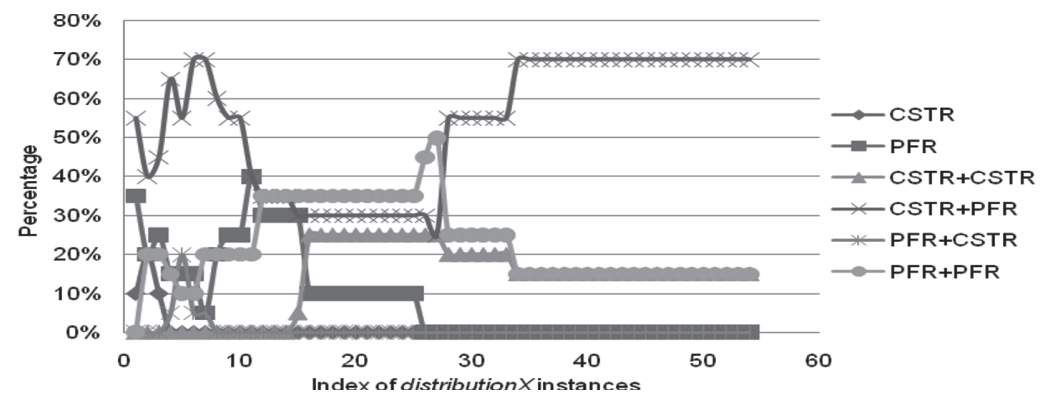

Figure 3 percentages of promising solutions with different $r_{1}+r_{2}$ sequences

Table 1 Performance of Van de Vusse application with different optimization models

\begin{tabular}{|l|c|c|}
\hline Optimization model & Number of solutions $\boldsymbol{N}_{\boldsymbol{S}}$ & Final objective value $\boldsymbol{y}^{\boldsymbol{b}}$ \\
\hline Ontology-supported & 589 & 3.6266 \\
\hline Original & 1509 & 3.6567 \\
\hline
\end{tabular}

\section{Conclusions}

By studying Van de Vusse case, the ontology-supported optimization model has proven that it can not only accelerate optimization search, but also explore and present new knowledge visually. Also automation of the ontology-supported optimization model can be achieved by designing a proper ontology and Java application.

\section{References}

Achenie L. K. E. and Biegler L. T., (1990) Superstructure based approach to chemical reactor network synthesis, Computers and Chemical Engineering, 14-23

Du D., Yang S., Kokossis A. C. and Linke P., (2007) Experience on gridification and hyperinfrastructure experiments in optimization and process synthesis, 17th European Symposium on Computer Aided Process Engineering, Bucharest, Romania

Gruber T. R., (1993) “A translation approach to portable ontology specifications”, Knowledge acquisition, 5, 199-220

Kokossis A. C. and Floudas C. A., (1990) "Optimization of complex reactor network - I. Isothermal operation”, Chemical Engineering Science, 45(3): 595-614

Labrador-Darder C., (2009) "Semantically enabled process synthesis and optimization", PhD Thesis, Chemical and Process Engineering, Faculty of Engineering and Physical Sciences, University of Surrey

Marcoulaki E. C. and Kokossis A. C., (1996) "Stochastic optimization of complex reaction systems", Computers and Chemical Engineering, 20: S231-S236

Mehta V. L. and Kokossis A. C., (2000) "Nonisothermal synthesis of homogenous and multiphase reactor networks", AIChE Journal, 46(11): 2256-2273

Neches R., Fikes R. E., Finin T., Gruber T. R., Senator T., Swartout W. R., (1991) "Enabling technology for knowledge sharing", AI Magazine, 12(3), 36-56

Yang S., (2009) "On the Development of SA Cascade Optimization Algorithm - Application to Reactor Network Synthesis and Distributed Computing", PhD Thesis, Chemical and Process Engineering, Faculty of Engineering and Physical Sciences, University of Surrey

Yang S., Kokossis A. C. and Linke P., (2006) "Toward a novel optimization approach with simultaneous knowledge acquisition for distributed computing environments", ComputerAided Chemical Engineering, 21A: 327-332 